\title{
Making optics appealing in Colombia through low-cost experiments with lasers
}

Juan Álvarez, Nicolás Barbosa, Sergio Cotrino, David Guzmán, Víctor Mahecha, et al.

Juan R. Álvarez, Nicolás Barbosa, Sergio Cotrino, David A. Guzmán, Víctor Mahecha, Cristian Medina, M. Cristina Navarrete, Leonardo Uribe, Alejandra Valencia, "Making optics appealing in Colombia through low-cost experiments with lasers," Proc. SPIE 9793, Education and Training in Optics and

Photonics: ETOP 2015, 979333 (8 October 2015); doi: 10.1117/12.2223254

Event: Education and Training in Optics and Photonics: ETOP 2015, 2015, Bordeaux, France 


\title{
Making optics appealing in Colombia through low-cost experiments with lasers \\ Juan R. Álvarez, Nicolás Barbosa, Sergio Cotrino, David A. Guzmán*, Víctor Mahecha, Cristian Medina, M. Cristina Navarrete, Leonardo Uribe, Alejandra Valencia Physics Department, Universidad de los Andes, A.A. 4976, Bogotá D.C., Colombia
}

\begin{abstract}
With the aim of making optics reachable to all audiences, regardless of their age or area of study, we have decided to select, build and test a set of four experiments based on optical phenomena. An important factor in our approach is that the experiments should be used by any non-experienced exhibitor to amaze the audience and to arise in them interest in optics. Ease of setup is therefore desired. Requirements such as durability, repeatability and reduced cost are welcome as well.

Taking advantage of the low prices of laser pointers, we focused on experiments which use this nowadays accessible element. The experiments that integrate our selection, costing less than USD75, are: a water stream optical fiber, curved light beams on a honey-water mixture, an optical music transmitter-receiver, and holographic film projections. Among the covered concepts are reflection and refraction of light, color, optical communications, optical interference and modern everyday life's applications.

We have presented these setups in activities at our university to a wide range of educational levels, from 12-year old students, passing by last year high school students on a career day event, not leaving behind undergraduate students of any discipline. Moved by the positive response of the audience, we plan to expand its application to continuing education courses and kids' science fairs. We proved that having low-cost setups, useful when teaching science in developing countries, can help to broaden the target audience.
\end{abstract}

Keywords: education in optics, low-cost experiments, lasers, outreach

\section{INTRODUCTION}

One of the aims in educational physics is to teach how nature works, and made a relevant understatement of physics to make the learning process easy and intuitive. A simple way to do this, is to show the students the phenomena in real live situations in order to create an own image, leading to understand the topics easier. This kind of demonstrative experiments should be simple in a way that any student can understand it. If the student feels some interest on it, ideally the student should acquire the necessary skills to recreate it, in such a way that this knowledge is seeded to be transmitted in a future.

The present research is a compilation of four demonstrative experiments that the OSA student chapter at Universidad de los Andes in Colombia have designed and tested for an audience with a low level of physics knowledge. Each experiment shows a different physical phenomenon and tries to generate an impact in the public in order to generate curiosity.

We made an effort in reducing costs, to provide the audience the possibility to reproduce them without making an important investment. Also special attention was put in the naming process of the experiments, so people get interest on it and want to see it performed to understand what it is about. The four experiments we present here are: optical fiber cascade, holographic film projections, curved light and optophonograph. In what follows each experiment is described.

\section{OPTICAL FIBER CASCADE}

The objective of this experiment is to grab the attention of the public in the working mechanism of the optical fiber in an interesting and simple way. The basic principle of this experiment is based on the total internal reflection property of light. It is widely known that Snell's law ${ }^{1}$ describes the behavior of light as it changes from one medium to another.

*da.guzman35@uniandes.edu.co

Education and Training in Optics and Photonics: ETOP 2015, edited by Eric Cormier, Laurent Sarger Proc. of SPIE Vol. 9793, 979333 - @ 2015 SPIE, IEEE, OSA, ICO · doi: 10.1117/12.2223254 
Each medium has an intrinsic property called the refraction index, which accounts for the collective velocity of light $\mathrm{v}$ in the medium with respect to its velocity in vacuum, c. Mathematically, the refraction index is expressed as $n=c / v$. When light comes from a medium of index $n_{1}$ to a medium of index $n_{2}$ with an angle $\theta_{1}$ with respect to the interface, the angle of refraction, $\theta_{2}$, is given by the following relationship:

$$
n_{1} \sin \theta_{1}=n_{2} \sin \theta_{2}
$$

However, it is known that when $\theta_{1}$ surpasses a certain value, there is no way in which the light can surpass the barrier. This angle is known as the critical angle, at which light is entirely reflected. Mathematically, this critical angle is

$$
\theta_{1}=\sin ^{-1}\left(\frac{n_{2}}{n_{1}}\right) .
$$

In optical fiber, the light is always totally reflected for a prolonged interval in space. This is widely used for the transmission of information via light, a principle that can be seen simply in the optical music transmitter-receiver. The advantage that optical fiber presents with respect to air transmission is especially the loss of information due to noise in air. Nowadays, optical fibers are used to transmit information in distances as long as the Atlantic Ocean.

\section{Experimental setup}

Our experiment is based in the principles discovered in the 19th century by Daniel Colladon. ${ }^{2}$ In his experiment he pointed a collimated source of light to a stream of water, in which the light propagated due to the principle explained before. In our experiment, our collimated source of light is a laser pointer, which costs about \$3 USD. A perforation is made in a recipient where water is stored, ensuring that the beam is directed towards this aperture. A scheme of our experiment is shown in figure 1. Finally, all water is recovered in a second recipient, ensuring the sustainability of the experiment. The cost of both recipients is about \$10 USD. Moreover, water does not need to be entirely clean or suitable for drinking.

Required materials are

Plastic pitcher - \$5 USD

Plastic container - \$5 USD

Laser pointer - \$3 USD

Water

This experiment drew a very positive result, as an outreach experiment in our university. The public acquired interesting information about the working mechanisms for information transmission, and found a purposeful use of basic science in their everyday lives.
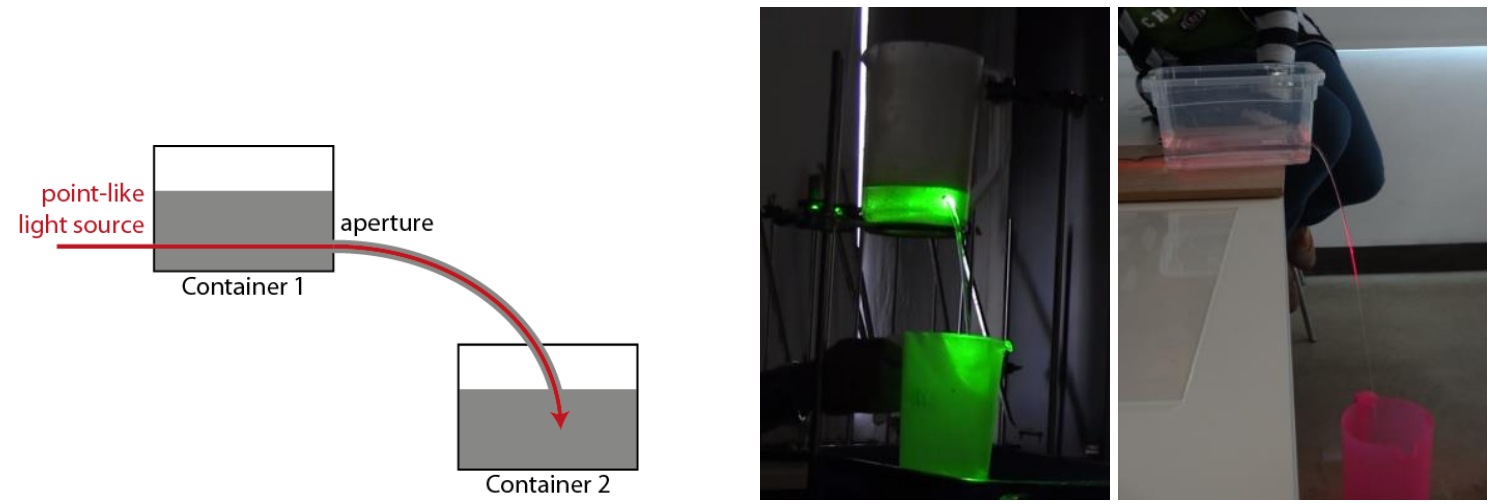

Figure 1. Left: schematic of the experimental setup of a water optical fiber cascade. Right: photographs of two of our implementations of a water optical fiber cascade with laser pointers. 


\section{HOLOGRAPHIC FILM PROJECTIONS}

Holography is a photography technique that allows producing three dimensional images. Although this technique is very similar to the traditional photography, it can't be considered a photo because the hologram contains a series of surface profiles that interact with light and creates the illusion of 3D, instead of a single image. The most common technique in holography is the transmission hologram that creates virtual images with a limited angle range. For a $360^{\circ}$ view, we use the full-view technique that changes the setup allowing a wider angle of observation, and then creating a 3D image on the film. ${ }^{3}$

The hologram is produced by a series of light interference patterns between the light source and the illuminated object, as shown in figure 2. The light used must be coherent and have the same phase, such as the one offered by a laser source. The full-view hologram is made with a photo sensible film rolled and placed around the object. The film and the object are illuminated by a laser using a divergent lens. The beam that goes directly to the film works as a reference beam while the reflection from the object carries the information, creating therefore the interference pattern. This procedure generates a virtual image of the object that can be seen at 360 degrees because the film will contain the information from all the perspectives.
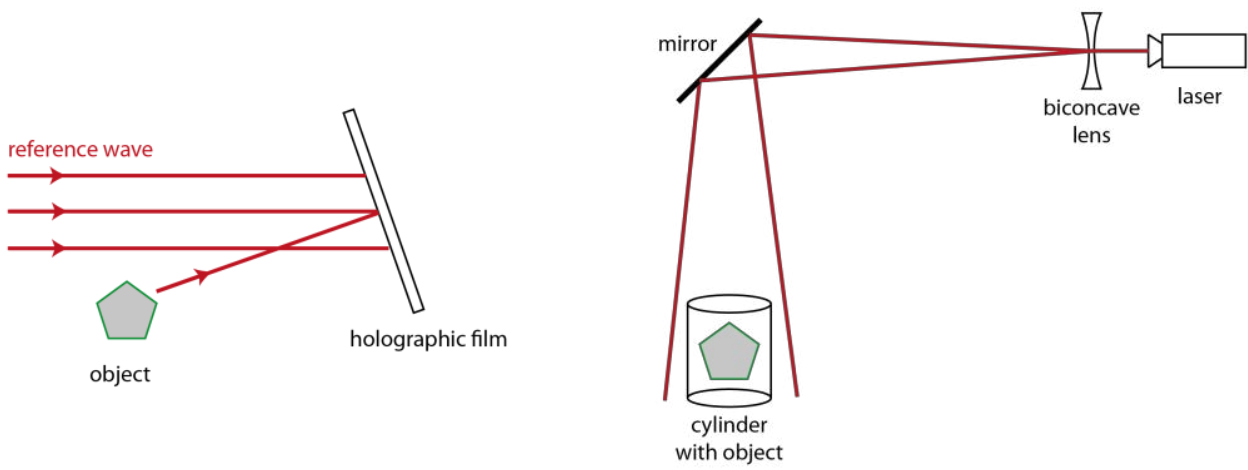

Figure 2. Schemes of the setups required to record a holographic image using the transmission technique (left) and the fullview technique (right).

\section{Experimental setup}

The hologram capturing process is better to be made over an optical table to avoid vibration and in a dark room preventing to overexpose the film. First, a helium-neon laser of wavelength $633 \mathrm{~nm}$ is send through a biconvex lens of focal distance $25.4 \mathrm{~mm}$. Then the beam is redirected to a cylinder that contains the object and the film with a layer of silver halide facing inside. At the bottom of the cylinder, there is a convex mirror that reflects the remaining light to the cylinder walls increasing the light intensity covering the film surface. A sketch of the assembly to record the holograms is shown in figure $2 .{ }^{4}$ The films used on the experiment have reference PFG-01 made by Intergraph LLC. They have a peak of light sensibility at $600-680 \mathrm{~nm}$ with a resolution of 3000 lines per $\mathrm{mm}$.

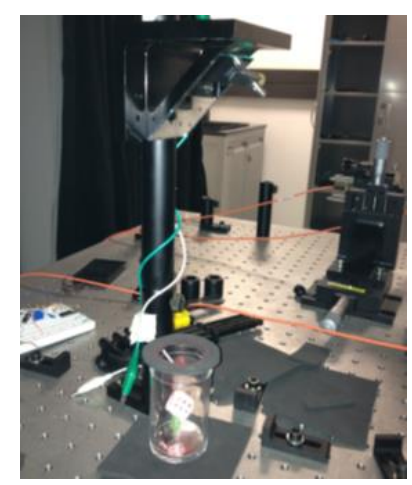

Figure 3. Setup used to record a full-view holographic image of a dice. Laser is outside the picture, which hits the mirror on the top of the image, and is redirected to the cylinder containing the object (dice). In the walls of the transparent cylinder is placed the holographic film. 

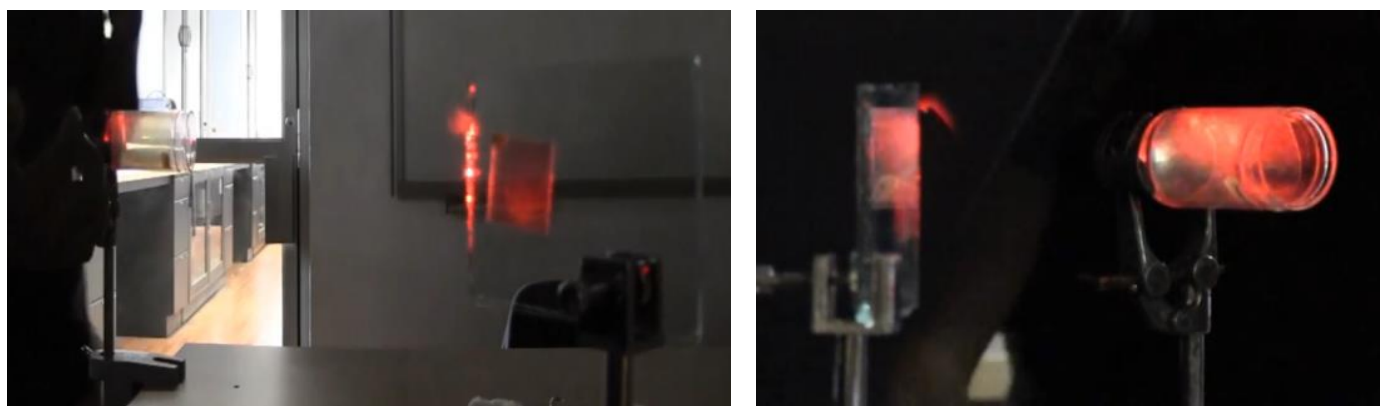

Figure 4. Setup used to reproduce a transmission hologram placed between two transparent plates (left) and a full-view hologram placed in a transparent cylinder (right).

\section{Film and reveal preparation}

Three chemical solutions are necessary to reveal the film.

a) Basic solution: to $750 \mathrm{ml}$ of distilled water add $20 \mathrm{~g}$ of catechol, $10 \mathrm{~g}$ of ascorbic acid, and $10 \mathrm{~g}$ of sodium sulphite and $75 \mathrm{~g}$ of urea.

b) Sodium carbonate solution: to $800 \mathrm{ml}$ of distilled water add $60 \mathrm{~g}$ of sodium carbonate.

c) Bleach solution: to $750 \mathrm{ml}$ of distilled water add $5 \mathrm{~g}$ potassium dichromate and $80 \mathrm{~g}$ of sodium bisulphate

Just before starting the reveal process mix the basic solution and the sodium carbonate solution, (it only lasts 8 hours). The reveal process is as follows:

1. Wash the film with the work solution for 2 minutes.

2. Wash on water, 3 min.

3. Soak on the bleach solution before the film gets transparent. 2 minutes approximately

4. Wash on water, 3 min.

5. Wash on photoflo and water

6. Let it dry

\section{Hologram projection}

The projection of the hologram is simpler than the capturing experimental assemble; the idea to recreate the hologram in the film is to apply the same light source used in the printing so it can be seen from outside the cylinder and at any angle. Additionally it is useful to be in a room with low or no additional light sources in order to have a better image of the projected object.

Materials and cost for the projection are

Holographic film- \$20 USD

Plastic cylinder - \$4 USD

Divergent diode laser - \$3 USD

\section{CURVED LIGHT}

This experiment intends to bend light by using a medium with a varying refractive index. With this experiment, it can be shown the basis of refractive index, Fermat's Principle and Snell's law. ${ }^{5}$ A material with variable refractive index can be regarded as a collection of very thin layers with a constant refractive index $n$. Then, when a light beam passes through all these layers, satisfies Snell's law and is refracted each time exposing a growing refraction angle. Performing this process several times, all these discontinuities are seen as a continuous curve. ${ }^{6}$ 


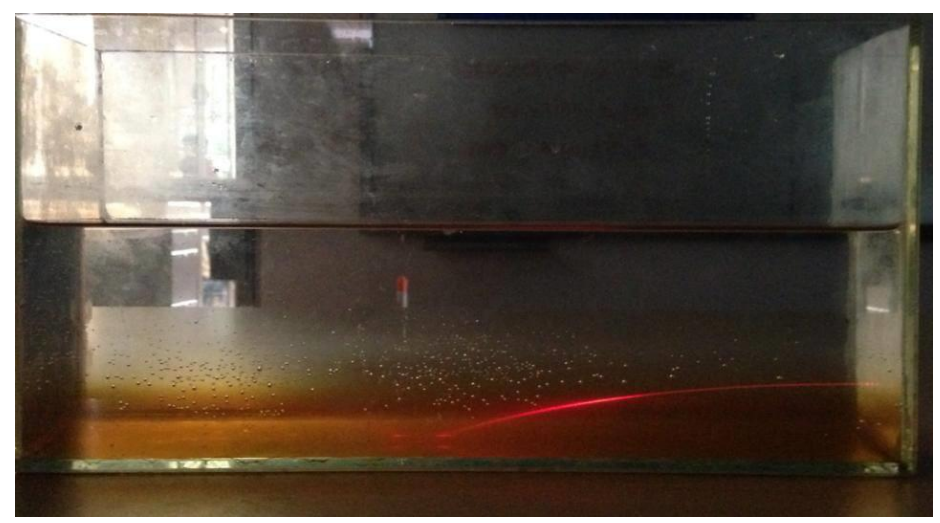

Figure 5. Curved light experiment. The honey-water mixture is illuminated with a red laser from right to left. A second arc is produced as the first arc is reflected on the bottom of the glass container, which can be slightly seen on the left of the picture.

\section{Experimental setup}

In this experiment we used honey because it is denser than water, sinks immediately into the container and then diffuses. This can also be achieved with other heavier-than-water fluid such as glycerin. The important fact is to use a substance that can diffuse progressively along the water contained in the recipient.

Required materials are

A translucent container - \$6 USD

Honey or corn syrup - \$7 USD

Water

To make the experiment, first fill the container with a desired height of water. Then pour honey into the container, so it creates at the bottom of the container a layer of about 2 or $3 \mathrm{~mm}$. Finally, allow the mixture stand at least 2 days. This will allow the honey in the bottom to diffuse into the water that is in the top, so it can be easily created a medium with varying refractive index (graded-index medium). A photograph of our implementation is shown in figure 6, where a laser is bended through the prepared mixture.

\section{OPTOPHONOGRAPH}

Communication is nowadays a high technology industry, and information traffic had become crucial in our life. A lot amount of data is shared along the globe almost instantaneously and continents have become interconnected by satellites and interoceanic optic fibers. ${ }^{7}$ Information can be carried now by digital or analog methods, and also by wired and wireless processes using electric signals, wave signals, light signals, audio, among others.
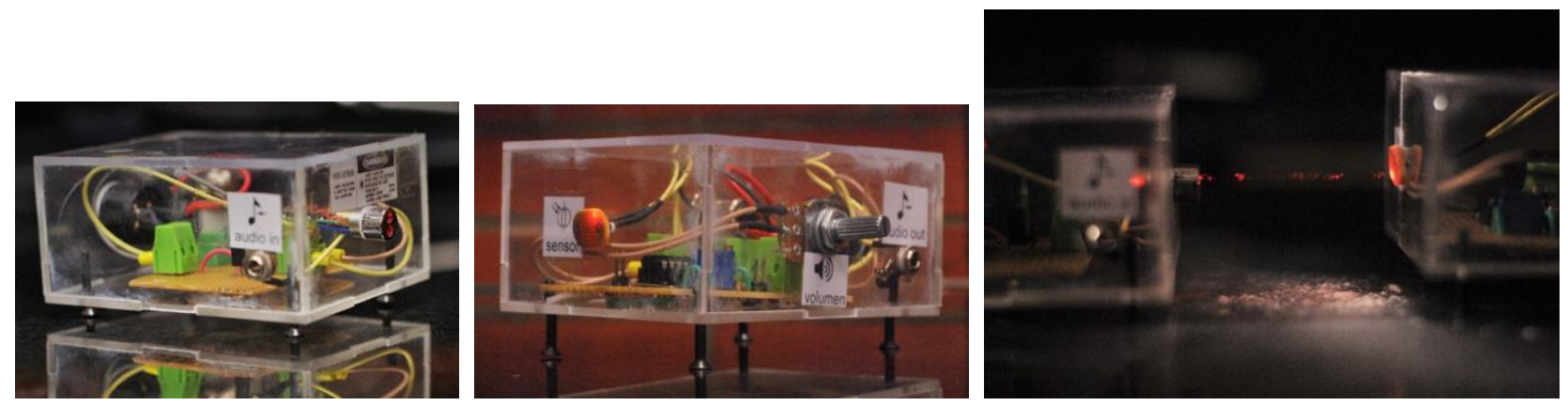

Figure 6. Pictures of our optophonograph. From left to right: a) transmitter, b) receiver, and c) transmitter sending signal to receiver. Signal enters the transmitter through an audio jack from any music player device, and alters the intensity of the laser. The receiver reads the laser signal with a photoresistor, and sends the electrical signal to an external speaker or earphones through an audio jack. Volume can be set in the receiver. 
Light is a good transmitter of information because of its wave's properties and its speed; there nothing faster! This makes light a perfect solution to carry information across long distances. The ways to transmit information have evolved through the pass of time. Specifically, the laser can be used to transmit information both in digital and analog formats.

The optophonograph is a different wireless way to transmit information using light, in this case through a laser. Compared to the common ones like the radio waves such as radio and cellphone networks, the main difference is that we can actually see the path of the transmitted information, which makes it appealing to the audience. Our implementation is an evolution of a previous proposal that originally requires a transformer ${ }^{8}$, which is eliminated to reduce costs and weight.

\section{Experimental setup}

The required materials are

Diode laser - \$3 USD

Electronic components - \$10 USD

Plastic box - \$12 USD (optional, but helps when aligning)

Audio cable - \$2 USD

Speaker - \$10 USD (optional, personal earphones might be used)

The optophonograph is a device composed by two parts: a receiver and a transmitter. The way to communicate these separated devices is by optical means using a laser. The receiver has a speaker attached to it and the music can be listened from there.

To achieve the communication between the separated devices (transmitter and receiver), both have to be aligned with the laser pointing from one to the other, as shown in figure 6. It is relevant to notice that there will be interferences caused by another light sources in the environment. The darker the room the clearer the sound will be.

The transmitter is connected to a regular power source and also it receives the music from a normal music player device. This analog signal coming as voltage is the one that makes the laser intensity to fluctuate, such that the laser transmits the information that it obtains from the music player.

The receiver has a photoresistor which receives the signal from the transmitter, converting the optical signal into an electrical one. This signal is processed via another circuit like the one in the transmitter (but the opposite). Finally the music can be listened with the speaker or earphones.

\section{DISCUSSION AND CONCLUSIONS}

The most satisfying feeling is to see the audience's interest about every topic related to what we showed to them. During each experiment we can see if everything is going fine by the smiles on the faces of all the people that is watching us; they are amazed and that is what any teacher would want. The audience typically wants to know everything about the experiment, noticed when they ask about why each experiment works and what is the physics behind. These experiments are not very complicated or especially uncommon to what we see daily, but perhaps nobody has shown students in this appealing way, which explains why everyone gets surprised.

Our main objective is to reach to every person regardless of their area of study or age, and we can say that this objective is accomplished. Everyone is curious enough to be enhanced into learning about the physics behind each experiment. From our experience with these settings, we suggest not to get sophisticated about the theory because some people may not understand anything and also they will get bored. 

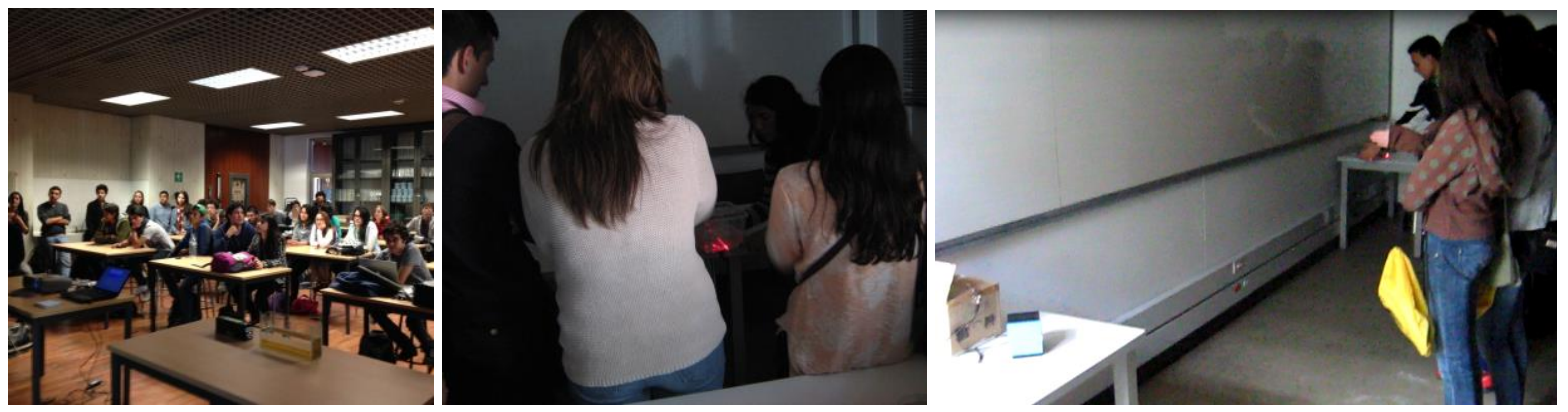

Figure 7. Sessions of students watching demonstrations of some of the described setups. Left: curved light, placed in front table; center: optical fiber cascade shown to high school students; right: the optophonograph where transmitter (back) and receiver (front) are in separated tables.

As already stated, since the setups of the experiments are costing less than \$75 USD in total, if someone on the audience is interested on recreating the experiment by them, there is no problem; almost all the materials are easy to find on Colombia's market and are inexpensive. In our experience, these experiments are recognized in the scientific community but are unknown to a general audience. In some occasions people from the audience explains to us that they have seen a similar experiment on Internet, but it is totally different to have the experience of watching them live. The activities are intended to be interactive, and accompanied by an explanation of the physical phenomena in an easy way to understand, something that a video can't show.

\section{REFERENCES}

[1] Hecht, E., [Óptica] Addison-Wesley Iberoamericana, Madrid (2000).

[2] Hecht, J., [City of Light: The Story of Fiber Optics] Oxford University Press, New York (1999).

[3] Saxby, G., [Practical Holography, 3rd edition], CRC Press, Boca Raton (2003).

[4] Industrial Fiber Optics, [Sandbox Holography Kit], Industrial Fiber Optics, Tempe (2014).

[5] Argáez-Mendoza, S., Oliva, A.I., "Fermat's principle, the branquistochrone, and the light's curve trajectory?," Ingeniería 15 (1), 47-55 (2011).

[6] Träger, F., [Springer Handbook of Lasers and Optics] Springer Science \& Business Media, New York (2007).

[7] Zeng, D., [Applied Informatics and Communication, Part I: International Conference, ICAIC 2011, Xi'an, China, August 20-21, 2011], Springer-Verlag, Berlin Heidelberg, (2011).

[8] Alonso, B., Hernández, C., [El láser, la luz de nuestro tiempo], Globalia Anthema, Salamanca, 196-198 (2010). 